\title{
Evaluating the performance of one-dimensional chaotic maps in the network-on-chip mapping problem
}

\author{
Golnar Gharooni-fard ${ }^{1}$, Ahmad Khademzade ${ }^{2}$, \\ and Fahime Moein-darbari ${ }^{1 a}$ ) \\ ${ }^{1}$ Department of Computer Science, Islamic Azad University, Arak branch, Iran \\ ${ }^{2}$ Iran Telecommunication Research Center, Tehran, Iran \\ a)fahimeh_md@iau-arak.ac.ir
}

\begin{abstract}
Mapping is one of the most critical issues in designing a NoC-based system. A good mapping of an application to a NoC will lead to more traffic among resources, which are physically close on the chip. In this paper, we introduce several one-dimensional chaotic maps for solving the $\mathrm{NoC}$ mapping problem. In addition we compare the solution qualities in accordance with different criteria mainly communication cost and convergence time. The results confirm an increase, due to chaotic sequences, in the value of some performance indexes.
\end{abstract}

Keywords: network-on-chip, mapping, chaotic maps

Classification: Integrated circuits

\section{References}

[1] A. Jantsch and H. Tenhunen, Networks on chip, Kluwer Academic Publishers, Dordrecht. USA, 2003.

[2] F. Moein-darbari, A. Khademzade, and G. Gharooni-fard, "CGMAP: A New Approach to Network-on-Chip Mapping Problem," IEICE Electron. Express, vol. 6, no. 1, pp. 27-34, Jan. 2009.

[3] W. J. Dally and B. Towels, "Route Packets, Not Wires: On-Chip Interconnection Networks," Proc. DAC'01, New York, USA, pp. 684-689, 2001.

[4] S. Kumar and A. Jantsch, "A Network on Chip Architecture and Design Methodology," Proc. VLSI'02, Pittsburgh, Germany, pp. 105-112, 2002.

[5] T. Lie and S. Kumar, "Two-step Genetic Algorithm for Mapping Task Graphs to Network on Chip Architecture," Proc. DSD'03, pp. 180-187, Sept. 2003.

[6] S. Murali and G. D. Micheli, "Bandwidth-Constrained Mapping of Cores onto NoC Architectures," Proc. DATE'04, vol. 2, pp. 896-901, Feb. 2004.

[7] W. T. Shen, C. H. Chao, Y. K. Lien, and A. Y. Wu, "A new binomial mapping and optimization algorithm for reduced-complexity mesh-based on-chip-network," Proc. 1st International Symposium on Network-onChip (NOCS'07), June 2007.

[8] Peter Stavroulakis, Chaos Application in Telecommunications, CRC press Taylor \& Francis, Boca Raton London New York, 2006. 
[9] M. Bucolo, R. Caponetto, L. Fortuna, M. Frasca, and Rizzo, "Does chaos work better than noise?," IEEE Circuits Syst. Mag., vol. 4, no. 3, pp. 419.

[10] E. Ott, Chaos in Dynamical Systems, Cambridge University Press, Cambridge, UK, 2002.

[11] A. Erramilli, R. P. Singh, and P. Pruthi, "Modeling packet traffic with chaotic maps," Royal Institute of Technology, Stockholm-Kista, Sweden, Aug. 1994.

[12] M. S. Tavasoei and M. Haeri, "Comparison of one-dimensional chaotic search pattern in chaos optimization algorithms," Elsevier journal of Applied Mathematics and Computation 187(2007), pp. 1076-1085, 2007.

\section{Introduction}

In the past decade, continuous improvements have been made in the semiconductor technology. This means that a whole processing system comprising processors, memories, accelerators, peripherals, etc. can now be integrated in a single silicon die. The term Network on Chip (NoC) is today used mostly in a very broad meaning, encompassing the hardware communication infrastructure, the middleware and operating system communication services and a design methodology and tools to map applications onto a NoC. All this together can be called a NoC platform [1]. The Network-on-Chip platform provides the communication infrastructure for the resources. In this way it is possible to develop the hardware of resources independently as standalone blocks and create the NoC by connecting the blocks as elements in the network.

There are many different ways to design a NoC, depending on the network architecture and protocol choice. We choose a two dimensional mesh interconnection, which is very simple from a layout perspective, and the local interconnections between resources and switches are independent of the size of the network.

Since the mapping problem is an NP-hard one, we attempt to use a technique to obtain an optimal approximation of the desired performance indexes (delay, power consumption, etc.). The Chaos-Genetic based Algorithm (CGMAP) presented in [2], seems to have the potential to achieve this goal very well. Therefore it is used as a solution for the mapping problem in this work.

\section{Related work}

NoC mapping problem has become a broad topic of research and development in the new millennium. In [3], Dally and Towels, use the on-chip network instead of ad hoc global wiring to interconnect the entire IP module on a chip. In [4] Kumar and Jantsch, implement a 2D mesh NoC architecture that consists of a mesh of IP cores with an associated router for each core. In order to optimize the results of the mapping problem, various people try to enhance the results considering different performance elements. Lie and 
Kumar, proposed a two-step Genetic algorithm for mapping task graphs to the NoC architecture in [5], with the objective of minimizing the average communication delay of the network.

Fallowing the same objective in [6] Murali and De Micheli, proposed NMAP, a fast algorithm that maps the cores onto mesh NoC architecture under bandwidth constraints, which could be extended to map cores onto different NoC topologies. BMAP is a binomial mapping and optimization algorithm presented in [7], to reduce the hardware cost of on-chip network infrastructure. BMAP is proved to have less computational complexity compared to the existing mapping algorithms.

\section{The NoC mapping problem}

The input of the NoC mapping problem is a directed task graph $G(V, E)$ in which every $v_{i} \in V$ denotes a processing element or a memory unit (generally an IP core), and a directed edge $e_{k}=\left(v_{i}, v_{j}\right)$ denotes a communication trace from the source node $v_{i}$ to the destination node $v_{j}$. The weight of the edges $w\left(e_{k}\right)$ usually refers to the communication cost between two corresponding nodes.

A mesh-based topology of $\mathrm{NoC}$ is defined by $T(U, L)$ where each vertex $u_{i} \in U$ denotes a node in the topology and each $l_{i} \in L$ denotes a physical link between two vertices. The weight of a link $w\left(l_{k}\right)$ represents the bandwidth available across the link $l_{k}$.

Recently, the idea of using chaotic systems instead of random processes has been noticed in several fields. One of these fields is optimization theory. In random-based optimization algorithms, the role of randomness can be played by chaotic dynamics, in order to possess a joint advantage of GA and the chaotic variable [3]. As stated earlier, the problem is to find a mapping of the vertices of a task graph to the tiles of a mesh based NoC architecture, with an objective of minimizing the communication cost between the IP cores.

\section{Chaos}

Chaos is a none-periodic, long-term behavior in a deterministic system that exhibits sensitive dependence on initial conditions. Edward Lorenz irregularity in a toy model of the weather displays first chaotic or strange attractor in 1963. It was mathematically defined as randomness generated by simple deterministic systems. A deterministic structure can have no stochastic (probabilistic) parameters. Therefore chaotic systems are not at all equal to noisy systems driven by random processes. The irregular behavior of the chaotic systems arises from intrinsic nonlinearities rather than noise.

In general, the most important defining property of chaotic variables is Sensitive dependence to Initial Conditions (SIC), which requires that trajectories originating from very nearly identical initial conditions diverge at an exponential rate. Pseudo-randomness and ergodicity are other dynamic characteristics of a chaotic structure [8]. The latter ensures that the track of a chaotic variable can travel ergodically over the whole space of interest. 
The variation of the chaotic variable has a delicate inherent rule in spite of the fact that it looks like a disorder.

\subsection{One-dimensional chaotic maps}

One-dimensional noninvertible maps are the simplest systems with capability of generating chaotic motion. They're capable of giving simple finite solutions without any complications. Here, we give a brief introduction to some wellknown one-dimensional chaotic maps.

\subsubsection{Logistic map}

In 1976 Robert May, a professor of biology at Princeton, pointed out that the logistic map led to chaotic dynamics. Logistic map is a polynomial mapping of degree 2. It is often cited as an example of how complex behavior can arise from a very simple nonlinear dynamical equation. This map is defined by [9]

$$
x_{n+1}=f\left(\mu, x_{n}\right)=\mu x_{n}\left(1-x_{n}\right) \quad, \quad 0<\mu \leq 4
$$

in which $\mu$ is a control parameter, and $x$ is the variable. Since (1) represents a deterministic dynamic system, it seems that its long-term behavior can be predicted. But the behavior of system in (1) is greatly changed with the variation of $\mu$. The value of the control parameter, determines whether $x$ converges to a constant point, oscillates between two or more values, or whether $x$ behaves chaotically in an unpredictable pattern.

\subsubsection{Tent map}

In mathematics, the tent map is an iterated function, in the shape of a tent, forming a discrete-time dynamical system. It takes a point $x_{n}$ on the real line and maps it to another point [10]:

$$
x_{n+1}=\left\{\begin{array}{cc}
\mu x_{n} \quad, \quad x_{n}<\frac{1}{2} \\
\mu\left(1-x_{n}\right), \quad \frac{1}{2} \leq x_{n}
\end{array}\right.
$$

where $\mu$ is a positive real constant. The tent map and the logistic map are topologically conjugate, and thus the behavior of the two maps is in this sense identical under iteration. Depending on the value of $\mu$, the tent map demonstrates a range of dynamical behavior ranging from predictable to chaotic.

\subsubsection{Bernoulli shift map}

The Bernoulli shift map belongs to class of piecewise linear maps which consist of a number of piecewise linear segments. This map is a particularly simple example, consisting of two linear segments to model the active and passive states of the source. It is defined as follows [11]:

$$
x_{n+1}=\left\{\begin{array}{cc}
\frac{x_{n}}{(1-\lambda)}, \quad 0<x_{n}<(1-\lambda) \\
\frac{x_{n}-(1-\lambda)}{\lambda},(d \equiv 1-\lambda)<x_{n}<1
\end{array}\right.
$$


The Bernoulli shift, like all chaotic systems, starts with an initial parameter, transforms this parameter, and then uses this transformed parameter for the input of the next iteration.

\subsubsection{Sine map}

The Sine map is described by the following equation:

$$
x_{n+1}=\frac{a}{4} \sin \left(\pi x_{n}\right)
$$

where $0<a \leq 4$. This map has qualitatively the same shape as the logistic map. Such maps are called unimodal maps.

\subsubsection{ICMIC map}

The iterative chaotic map with infinite collapses (ICMIC) is defined by

$$
x_{n+1}=\sin \frac{a}{x_{n}}
$$

and $a \in(0, \infty)$ is an adjustable parameter.

\section{Experimental results}

In this section, we present the results of the execution of the Chaos-Genetic mapping algorithm on a benchmark application, Video Object Plane Decoder (VOPD) with 16 IP-cores and 20 links. Fig. 1. shows the core graph for this benchmark application. This application has also been used in [6] in order to evaluate the performance of the NMAP algorithm.

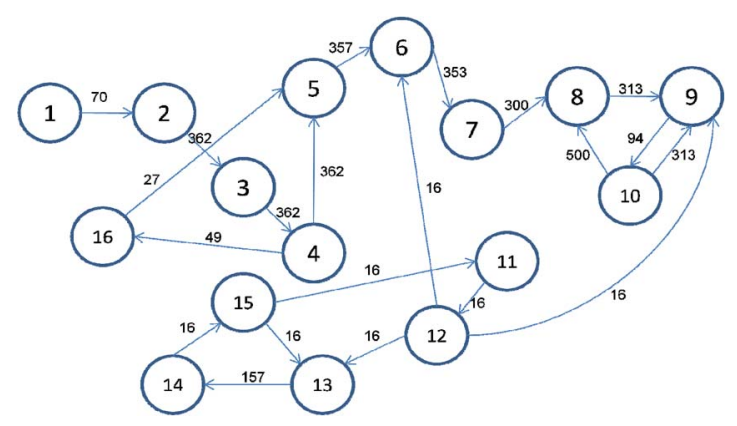

Fig. 1. Core graph of VOPD

Tavazoei and Haeri [12], presented a Comparison of different onedimensional maps as chaotic search pattern in chaos optimization algorithms. In this section we compare the efficiency of the discussed chaotic maps as the mapping operator in CGMAP algorithm. Since the algorithm is mainly based on genetic algorithms, therefore different results may be obtained each time it is executed. We use the average result obtained after 20 times of executing the algorithm using a particular chaotic map as a mapping operator. Each column in Fig. 2 (a) represents the rate of convergence to the best solution for the specified map. The benchmark application used in this section is 
VOPD. It is noticeable that, ICMIC and Tent map has the maximum convergence rates among the others and the minimum convergence rate belongs to Bernoulli Shift and Logistic maps. This means that ICMIC map works very well as a chaotic operator, and can achieve acceptable solutions within the shortest time.

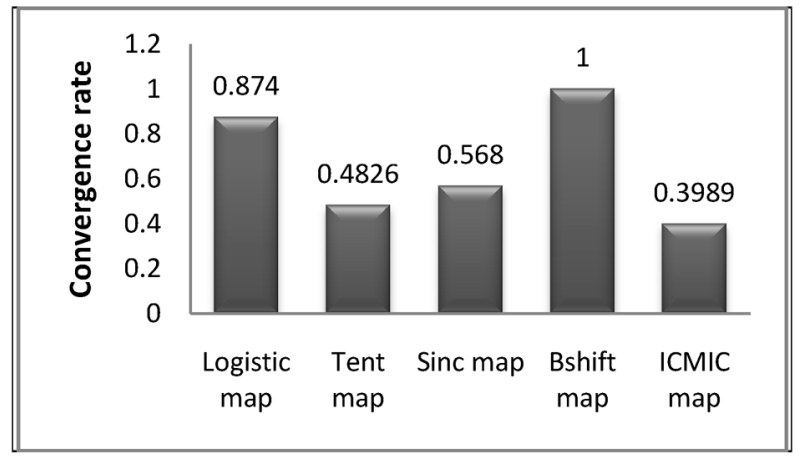

(a)

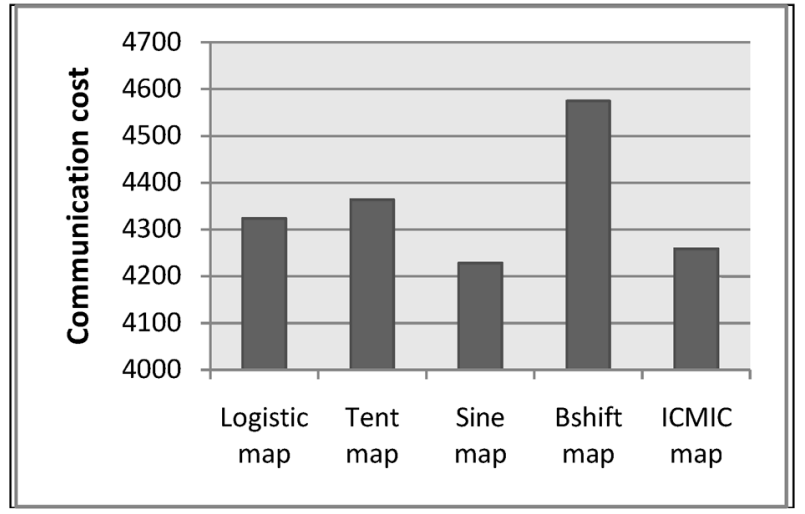

(b)

Fig. 2. (a): Rate of convergence to the best solution for each chaotic map applied on VOPD (b): Comparison between the communication costs of chaotic maps applied on VOPD

We also compare the communication costs of executing the algorithm using each of the discussed chaotic maps and demonstrate the average results in Fig. 2 (b). As it is clear Sine map achieves the lowest communication cost among the others and Bernoulli Shift on the other hand, gains the worst result. Although the choice of the most effective chaotic map is extremely dependable on the benchmark problem, we usually need to make a tradeoff between the convergence rate and communication costs.

Furthermore, we've calculated the time complexities of the mapping algorithm using all the mentioned chaotic maps. Results made it clear that the complexity of the mapping algorithm does not change much when using different chaotic equations. As it is proved in [2], the complexity of the mapping algorithm is equal to

$$
O\left(k\left(c \times e \times n^{2}\right)\right)
$$


Where $n$ is the number of nodes in the task graph, $e$ is the number of edges in the task graph, and $c, k$ are the constants related to the GA's design defining the population size and the number of generations. Eq. (6) holds for all the five chaotic maps. In other words, time complexity of the mapping algorithm is independent of the chaotic equation used during the implementation process.

\section{Conclusion}

In this paper we evaluate the effectiveness of the chaotic maps in reaching the best solution for the NoC mapping problem using a chaos-genetic based algorithm. Five most commonly used one-dimensional chaotic maps are introduced and the communication cost and the convergence rate for each map is calculated.

The experiments carried out on a real application (VOPD) made it possible to compare the performance of chaotic equations in finding an acceptable solution for the NoC mapping problem. The Results obtained from using each chaotic map differed with the others in view of convergence rate and communication cost. Therefore, by prioritizing the favorites that one seeks, a chaotic equation may be selected that is the most congruent with ones will. Albeit, choosing the best map for optimizing is a problem-based subject. That means none of these chaotic maps transcends other maps for all problems and for all desired criteria. 